\title{
Poor People and Poor Health: Examining the Mediating Effect of Unmet Healthcare Needs in Korea
}

\author{
Youngsoo Kim, Saerom Kim, Seungmin Jeong, Sang Guen Cho, Seung-sik Hwang \\ Department of Preventive Medicine, Graduate School of Public Health, Seoul National University, Seoul, Korea
}

Objectives: The purpose of this study was to estimate the mediating effect of subjective unmet healthcare needs on poor health. The mediating effect of unmet needs on health outcomes was estimated.

Methods: Cross-sectional research method was used to analyze Korea Health Panel data from 2011 to 2015, investigating the mediating effect for each annual dataset and lagged dependent variables.

Results: The magnitude of the effect of low income on poor health and the mediating effect of unmet needs were estimated using age, sex, education level, employment status, healthcare insurance status, disability, and chronic disease as control variables and selfrated health as the dependent variable. The mediating effect of unmet needs due to financial reasons was between $14.7 \%$ to $32.9 \%$ of the total marginal effect, and $7.2 \%$ to $18.7 \%$ in lagged model.

Conclusions: The fixed-effect logit model demonstrated that the existence of unmet needs raised the likelihood of poor self-rated health. However, only a small proportion of the effects of low income on health was mediated by unmet needs, and the results varied annually. Further studies are necessary to search for ways to explain the varying results in the Korea Health Panel data, as well as to consider a time series analysis of the mediating effect. The results of this study present the clear implication that even though it is crucial to address the unmet needs, but it is not enough to tackle the income related health inequalities.

Key words: Unmet healthcare needs, Needs assessment, Healthcare disparities, Korea Health Panel

\section{INTRODUCTION}

Donabedian proposed a conceptual model that provide a framework for examining healthcare, defining healthcare needs as "required medical services that can appropriately prevent, alleviate, and cure the status of illness or inability

Received: July 12, 2018 Accepted: January 23, 2019

Corresponding author: Saerom Kim, MD, MPH

Department of Preventive Medicine, Graduate School of Public Health, Seoul National University, 1 Gwanak-ro, Gwanak-gu, Seoul 08826,

Korea

E-mail: saerom.rb@gmail.com

This is an Open Access article distributed under the terms of the Creative Commons Attribution Non-Commercial License (http://creativecommons.org/licenses/by$\mathrm{nc} / 4.0 / /$ which permits unrestricted non-commercial use, distribution, and reproduction in any medium, provided the original work is properly cited. caused by some disturbance in health and well-being" [1-3]. According to this model, unmet needs for medical care refer to a situation where a lack of medical care leads to the failure of appropriate prevention, alleviation, and cure of illness or disability. Those who experience unmet needs are, by definition, likely to see deteriorating health conditions, which is supported by previous studies that demonstrated correlations between unmet needs and various proxy indicators, such as death, quality of life scale, and self-rated health [4-8].

Researchers have also explored various factors that may impact unmet needs. Assuming unmet needs as a proxy indicator of lack of access to healthcare, many previous studies found the correlation between unmet needs and low education level, low income, chronic disease, living alone, limited activities [9-12]. The factors associated with unmet needs are 
generally known to be associated with poor health, thus theoretically and empirically, unmet needs are believed to mediate or interact with the various factors that affect poor health. To sum up, unmet needs have been regarded as a proxy for healthcare accessibility that should be addressed on its own terms, but their mediating effects on poor health have rarely been studied.

The purpose of this study was to examine the mediating effect of unmet needs on poor health. In order to properly gauge the mediating effect of unmet needs, it is necessary to verify healthcare need as an independent variable and its fulfillment, followed by estimating unmet needs' mediating consequences for health. However, it was not possible to find resources with specific data on healthcare needs that may or may not be unmet. Therefore, we decided to use low income as a proxy indicator of health needs, since it is associated with higher health risk and susceptibility, poor health behavior, and a higher prevalence of diseases [13-15]. To sum up, this study investigated whether unmet healthcare needs due to financial reasons mediate poor health and if so, the magnitude of the mediating effect.

\section{METHODS}

Cross-sectional research method was used to analyze the Korea Health Panel data between 2011 and 2015, investigating the mediating effects for each annual dataset and the lagged dependent variables.

\section{Data}

The Korea Health Panel data are a reliable resource representing the status of Korea's national healthcare and medical environment that have been generated since 2008. The data include details on medical services and costs, as well as major health-related indicators. We obtained the self-rated health and unmet needs that is measured in adult population in the survey. Five years of data between 2011 and 2015 were used for analysis.

\section{Analytical Model and Variable Measurement Methods}

The analytical model was created by replacing "health need" in the Donabedian model with "low income" and the model is shown in Figure 1.

According to the Donabedian model, the mediating effect of unmet needs should be calculated by using health need events as the independent variable. However, since there is no available data on health need events, the variable was replaced by "low income," one of the major factors known to cause poor health. Low income was defined as the lowest quintile of adjusted household income, calculated by dividing monthly household income by the square root of the number of household members [16].

Unmet needs were considered as the mediator variable, defined as replies of "yes" to the survey question "Have you ever missed seeing a doctor and/or getting a medical check-up that was necessary during the last year? (Not including dental treatments and/or check-ups)". Cases where the unmet need was caused by "financial reasons (cost of treatment)" were an-

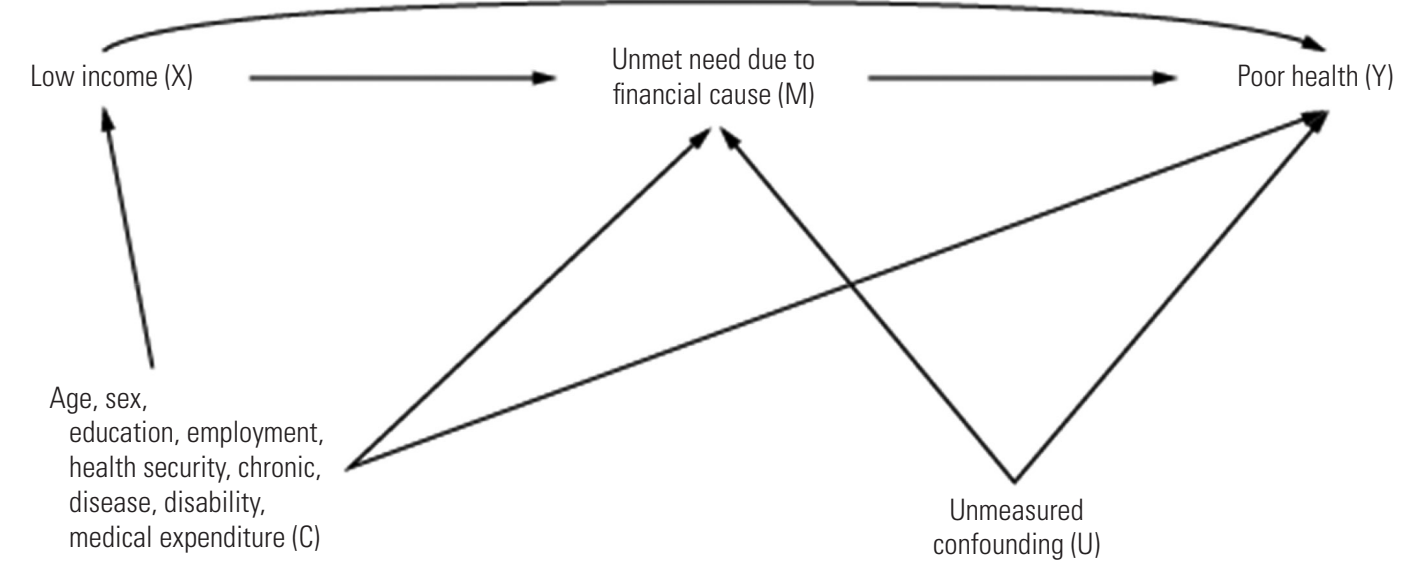

Figure 1. Analytical model for estimating the mediating effect of unmet needs. $X$, exposure variable; M, mediator; $Y$, outcome variable; $C$, confounding variables; $U$, unmeasured confounding. 
alyzed separately. Replies to the question on participants' selfrated health using a 5-point Likert scale of "normal", "good", "very good", "bad", and "very bad" were dichotomized, with the first 3 scores defined as "healthy" and the last 2 as "unhealthy". Self-rated health was used as an indicator to reflect the multifaceted quality of health [17-19].

The control variables included age, sex, education level (elementary school diploma and lower, high school diploma and lower, undergraduate and higher), employment status (fulltime regular employee, part-time employee, self-employed, and employer, unpaid family business, or unemployed), healthcare insurance status (National Health Insurance, Medical Aid), the presence of a disability or chronic disease, and survey year.

\section{Subjects of the Study}

The subjects of the Korea Health Panel surveys between 2011 and 2015 were adults aged 18 and older, and the composition of each panel is shown in Figure 2.

The subjects of the study were limited to adults aged 18 and older. Participants were excluded if data were missing from the additional questions that asked for variables such as selfrated health status and unmet needs; this was the case for $3.50 \%$ of the sample (2950 person-years). Responses that included the additional questions but had incomplete answers to questions related to self-rated health and unmet needs were found for $6.30 \%$ (4050 person-years) and $0.05 \%$ (33 per- son-years) of the observation, respectively. For healthcare insurance status, only 13 person-years was either not enrolled or in delinquency, thus this data was excluded from the study. An unbalanced panel consisting of 17761 participants (male: 8382; female: 9379) and 60779 person-years (male: 27542 person-years; female: 33237 person-years) was created.

\section{Statistical Analysis}

Unmet needs were confirmed to be correlated with poor health through a fixed-effects logit model in the results of a similar previous study [20], presented in Supplementary Material 1.

Quasi-Bayesian Monte Carlo estimation was used to estimate the mediating effects of unmet needs. This method allows estimation of not only the significance of mediating effects, but also the magnitude of the average causal mediation effect [2124]. We analyzed the Korean medical panel data cross-sectionally from 2011 to 2015 year by year. To estimate the mediation effect, a regression model with unmet needs as a dependent variable was used. The analytical model used is presented below:

logit $P$ (poor health $=1 \mid$ low income, unmet, covariates)

$=\theta_{0}+\theta_{1}$ low income $+\theta_{2}$ unmet $+\theta_{3}$ covariates $+\epsilon$

E(unmet | low income, covariates)

$=\beta_{0}+\beta_{1}$ low income $+\beta_{2}$ covariates $+\gamma$

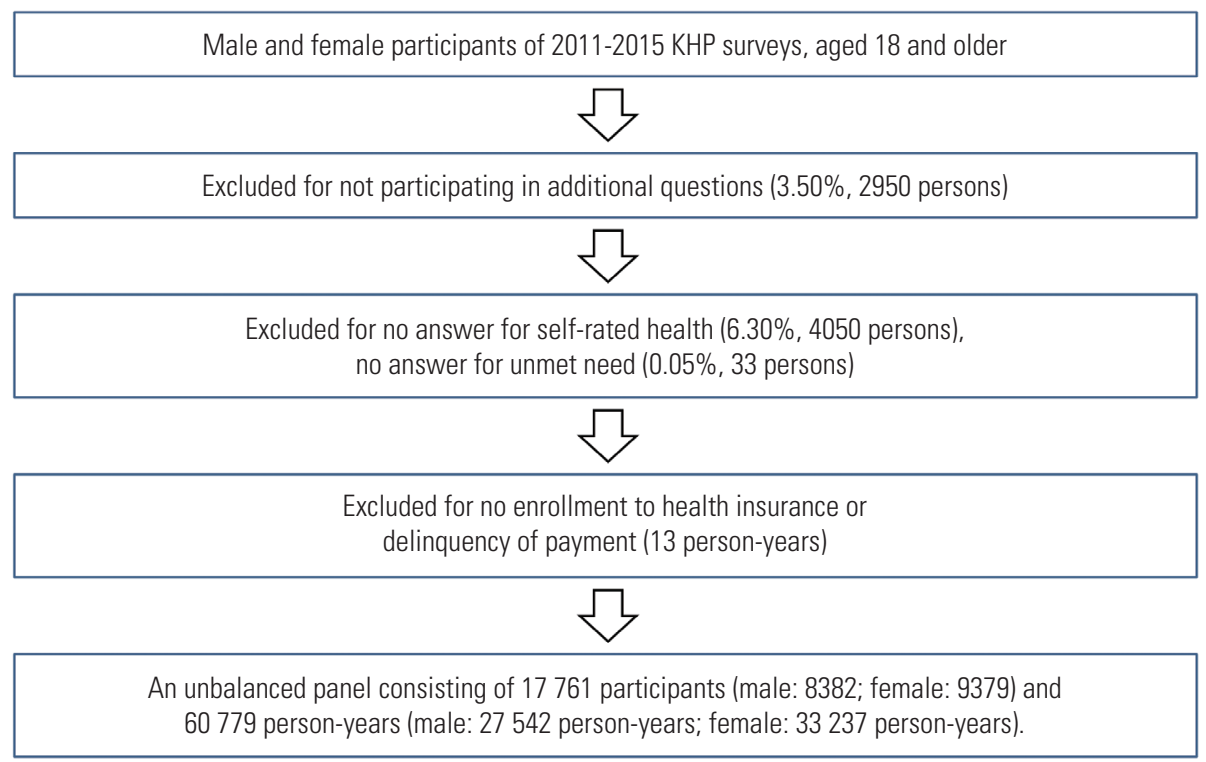

Figure 2. The composition of an unbalanced panel of subjects. KHP, Korea Health Panel. 
The lagged dependent variable analysis of the effects of unmet needs in year $t$ on health status in year $t+1$ was carried out considering the potential time lag between unmet needs and their actual effect on health. The analytical model is shown below:

logit $P$ (poor health ${ }_{t+1}=1 \mid$ low income ${ }_{t}$, unmet ${ }_{t}$, covariates $\left.{ }_{t}\right)$ $=\theta_{0}+\theta_{1}$ low income $_{t}+\theta_{2}$ unmet $_{t}+\theta_{3}$ covariates $_{t}+\epsilon$

$E$ (unmet $t_{t} \mid$ low income $t_{\text {, covariatest }}$ )

$$
=\beta_{0}+\beta_{1} \text { low income }_{t}+\beta_{2} \text { covariates }_{t}+\gamma
$$

In the analysis of mediating effects, income level was considered a relatively stable variable, while unmet needs may be different each year. Therefore, the controlled direct effects and the natural direct effects were both estimated, not considering the interaction between low income and unmet needs. The total marginal effects (TMEs) of low income on poor self- rated health, the direct effects of low income on health regardless of unmet needs, and the indirect effects of poor health mediated by unmet needs were estimated.

Stata version 15 (StataCorp., College Station, TX, USA) was used for the analyses, using the paramed and medeff commands for analyzing mediating effects.

\section{RESULTS}

\section{Characteristics of the Subjects}

The unbalanced panel included 11760 subjects in 2011, 7697 of whom were followed up for the next 5 years without missing data. New samples were added to the panel in 2014 to fill in attrition, which contributed to some changes in the characteristics and the trends of the subjects the following years. Participants who reported their self-rated health as poor accounted for $14.6 \%$ in $2011,16.0 \%$ in $2012,14.8 \%$ in 2013 , $16.7 \%$ in 2014 , and $14.8 \%$ in 2015 , respectively, demonstrat-

Table 1. General characteristics of the subjects

\begin{tabular}{|c|c|c|c|c|c|}
\hline Characteristics & $\begin{array}{c}2011 \\
(n=11760)\end{array}$ & $\begin{array}{c}2012 \\
(n=11026)\end{array}$ & $\begin{array}{c}2013 \\
(n=10539)\end{array}$ & $\begin{array}{c}2014 \\
(n=14028)\end{array}$ & $\begin{array}{c}2015 \\
(n=13426)\end{array}$ \\
\hline Poor self-rated health & $1714(14.6)$ & $1764(16.0)$ & $1559(14.8)$ & $2349(16.7)$ & $1986(14.8)$ \\
\hline Experienced unmet needs & $1764(15.0)$ & $1745(15.8)$ & $1833(17.4)$ & $1816(12.9)$ & $1872(13.9)$ \\
\hline Experienced unmet needs due to financial reasons & $529(4.5)$ & $624(5.7)$ & $579(5.5)$ & $482(3.4)$ & $531(4.0)$ \\
\hline Age $(y)$, mean $\pm S D$ & $51.0 \pm 16.5$ & $51.9 \pm 16.7$ & $52.5 \pm 17.0$ & $52.9 \pm 17.1$ & $53.5 \pm 17.4$ \\
\hline $18-64$ & $8856(75.3)$ & $8094(73.4)$ & $7554(71.7)$ & 9998 (71.3) & $9402(70.0)$ \\
\hline$\geq 65$ & $2904(24.7)$ & $2932(26.6)$ & $2985(28.3)$ & $4030(28.7)$ & $4024(30.0)$ \\
\hline Sex (male) & $5311(45.2)$ & $4978(45.1)$ & $4751(45.1)$ & $6389(45.5)$ & $6113(45.5)$ \\
\hline \multicolumn{6}{|l|}{ Education level } \\
\hline Elementary school diploma and lower & $2550(21.7)$ & $2409(21.8)$ & $2293(21.8)$ & $2933(20.9)$ & $2774(20.7)$ \\
\hline High school diploma and lower & $5144(43.7)$ & $4777(43.3)$ & 4508 (42.8) & 6010 (42.8) & $5709(42.5)$ \\
\hline Undergraduate and higher & 4066 (34.6) & 3840 (34.8) & $3738(35.5)$ & $5085(36.2)$ & $4943(36.8)$ \\
\hline \multicolumn{6}{|l|}{ Employment status } \\
\hline Full-time, regular employee & $2388(20.3)$ & $2192(19.9)$ & $2092(19.9)$ & $2753(19.6)$ & $2647(19.7)$ \\
\hline Part-time, temporary employee & $2132(18.1)$ & 2124 (19.3) & $1984(18.8)$ & $2770(19.7)$ & $2580(19.2)$ \\
\hline Self-employed, employer & $1958(16.6)$ & $1823(16.5)$ & $1761(16.7)$ & $2320(16.5)$ & $2012(15.0)$ \\
\hline Unpaid family business & $562(4.8)$ & $523(4.7)$ & $498(4.7)$ & $646(4.6)$ & $570(4.2)$ \\
\hline Unemployed & $4720(40.1)$ & 4364 (39.6) & $4204(39.9)$ & $5539(39.5)$ & $5617(41.8)$ \\
\hline Medical Aid (type 1, type 2) & $515(4.4)$ & $454(4.1)$ & $406(3.9)$ & $435(3.1)$ & $430(3.2)$ \\
\hline Prevalence of chronic disease & $7280(61.9)$ & $6922(62.8)$ & $6909(65.6)$ & $8920(63.6)$ & $8591(64.0)$ \\
\hline Disability & $777(6.6)$ & $742(6.7)$ & $687(6.5)$ & $901(6.4)$ & $855(6.4)$ \\
\hline $\begin{array}{l}\text { Household income (adjusted for number of members, } \\
10000 \mathrm{KRW}) \text {, median [IQR] }\end{array}$ & $\begin{array}{c}1918 \\
{[1210,2789]}\end{array}$ & $\begin{array}{c}1972 \\
{[1226,2934]}\end{array}$ & $\begin{array}{c}2061 \\
{[1282,3000]}\end{array}$ & $\begin{array}{c}2116 \\
{[1308,3141]}\end{array}$ & $\begin{array}{c}2250 \\
{[1370,3355]}\end{array}$ \\
\hline
\end{tabular}

Values are presented as number (\%).

SD, standard deviation; KRW, Korean won; IQR, interquartile range.

${ }^{1}$ The Medical Aid classifies beneficiaries into two categories, type 1 and 2, on the basis of being incapable (those under 18 or over 65 years of age, or disabled) or capable of working, respectively. 
ing no particular trend. The percentage of those who experienced unmet needs showed increasing trend from 2011 to 2013, then drop down from $17.4 \%$ in 2013 to $12.9 \%$ in 2014, which presumably indicate the effect of attrition and addition of new observations (Table 1).

\section{Primary Findings}

The TMEs, direct effects, and indirect effects of low income on self-rated poor health are presented as odds ratios (ORs) while the mediating effect of unmet needs was calculated as a percentage in Table 2, and the equations applied are shown below. The reference categories were high income (quintiles 2-5) for the direct effects, no unmet need for the indirect effects, and high income and no unmet need for the TMEs.

$$
O R^{T M E}=O R^{N D E} \times O R^{N E}
$$

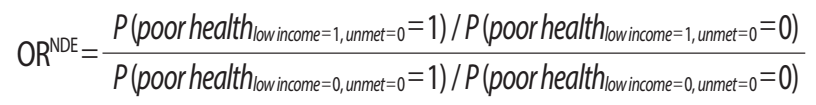

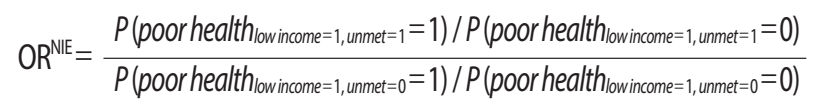

\begin{tabular}{|c|c|c|c|c|}
\hline Mediator variables & TME & NDE, CDE & NIE & $\%$ of total effect mediated \\
\hline \multicolumn{5}{|l|}{ KHP $2015(n=13426)$} \\
\hline Unmet needs & $1.51(1.33,1.70)$ & $1.43(1.26,1.60)$ & $1.06(1.04,1.08)$ & 13.5 \\
\hline \multicolumn{5}{|l|}{ KHP 2014 (n=14 028) } \\
\hline Unmet needs & $1.33(1.19,1.52)$ & $1.23(1.10,1.40)$ & $1.08(1.06,1.11)$ & 30.1 \\
\hline Unmet needs & $1.33(1.15,1.53)$ & $1.22(1.06,1.41)$ & $1.08(1.06,1.11)$ & 31.0 \\
\hline Unmet needs due to financial reasons & $1.34(1.16,1.54)$ & $1.22(1.05,1.40)$ & $1.10(1.07,1.13)$ & 32.9 \\
\hline \multicolumn{5}{|l|}{ KHP 2012 ( $n=11026)$} \\
\hline Unmet needs & $1.49(1.32,1.70)$ & $1.41(1.23,1.61)$ & $1.06(1.04,1.09)$ & 15.8 \\
\hline
\end{tabular}

Table 2. TMEs, direct effects, and indirect effects of low income on self-rated poor health

Values are presented as odds ratio (95\% confidence interval).

KHP, Korea Health Panel; TME, total marginal effect; NDE, natural direct effect; CDE, controlled direct effect; NIE, natural indirect effect.

\begin{tabular}{|c|c|c|c|c|}
\hline Mediator variables & TME & NDE, CDE & NIE & $\%$ of total effect mediated \\
\hline \multicolumn{5}{|l|}{ KHP 2014 (n=12 495) } \\
\hline Unmet needs & $1.34(1.18,1.52)$ & $1.28(1.13,1.45)$ & $1.05(1.03,1.06)$ & 16.1 \\
\hline \multicolumn{5}{|l|}{ KHP $2013(n=9667)$} \\
\hline Unmet needs & $1.36(1.18,1.56)$ & $1.30(1.14,1.49)$ & $1.04(1.02,1.07)$ & 14.4 \\
\hline Unmet needs & $1.44(1.25,1.66)$ & $1.39(1.21,1.60)$ & $1.04(1.02,1.06)$ & 11.5 \\
\hline Unmet needs due to financial reasons & $1.43(1.24,1.64)$ & $1.38(1.20,1.58)$ & $1.04(1.02,1.06)$ & 14.0 \\
\hline \multicolumn{5}{|l|}{ KHP $2011(n=10742)$} \\
\hline Unmet needs & $1.56(1.36,1.78)$ & $1.52(1.34,1.74)$ & $1.02(1.00,1.04)$ & 4.0 \\
\hline
\end{tabular}

Table 3.TMEs, direct effects, and indirect effects of low income on self-rated poor health in the following year

Values are presented as odds ratio (95\% confidence interval).

KHP, Korea Health Panel; TME, total marginal effect; NDE, natural direct effect; CDE, controlled direct effect; NIE, natural indirect effect; 
The results showed that the OR of TME of low income on poor health ranged from 1.33 in 2013 to 1.51 in 2015. Of all effects, those mediated by unmet needs were the lowest at $9.5 \%$ in 2011 and the highest at $31.0 \%$ in 2013. The magnitude of the TME was not very different in unmet needs due to financial reasons. The OR of TME of low income on poor health ranged from 1.31 in 2014 to 1.52 in 2015 in model using unmet need due to financial reasons as a mediator variable. The mediating effect of unmet needs due to financial reasons was mostly higher than the mediating effect of unmet needs with every reason, with the lowest effect seen in 2012 at $14.7 \%$ and the highest in 2013 at $32.9 \%$.

Table 3 demonstrates the results of lagged dependent variables analysis with a lag time of 1 year. The OR of TME of low income in a given year on the next year's poor health ranged from 1.34 in 2014 to 1.56 in 2011. The lowest percentage of effects mediated by unmet needs was seen in 2011 at 4.0\%, and the highest in 2014 at $16.1 \%$. The OR of TME of unmet needs due to financial reasons only range from 1.32 in 2014 to 1.55 in 2011. The lowest value of the mediating effect of unmet needs due to financial reasons was $7.2 \%$ in 2011 and the highest was 18.7\% in 2014.

\section{DISCUSSION}

This study sought to understand the correlation between unmet healthcare needs and poor health, as well as to estimate the extent of the contribution of unmet needs to poor health based on the 2011-2015 Korea Health Panel data. The magnitude of the mediating effect of low income on poor health was estimated in the mediating effect analysis, and the mediating effect of unmet needs due to financial reasons accounted for $14.7 \%-32.9 \%$ of the TME of income. The mediating effect of unmet needs due to financial reasons in the lagged dependent variables model of the following year's health was lower, at $7.2 \%-18.7 \%$. This result corresponds to the McKeown thesis [25] that one's health status is determined by various social factors more than the utilization of medical services, denying that medical intervention has the most significant impacts on health. The assumption that unmet needs have a considerable mediating effect on poor health among lowerincome people turned out to be only partially true based on the results of this study. The estimated values of the mediating effect of unmet needs, in turn, suggest that $67 \%$ to $85 \%$ of poor health in lower-income individuals cannot be resolved by simply meeting their healthcare needs.

In lagged variable model, during the analysis period, the mediating effect of unmet need due to financial reason increase from $7.2 \%$ to $18.7 \%$ while the mediating effect of unmet need due to whole reason increase from $4.0 \%$ to $16.1 \%$. And the mediating effect of economic reason is greater than the mediating effect of the whole reason stably by years. Considering these facts, this may be related to worsening economic inequality in Korea, diminishing purchasing power for medical services among low-income group. There is also a possibility that economic recession around 2010 may have had impacts on unmet needs and poor health with a time lag $[26,27]$. Also, the trend of an increasing mediating effect of unmet needs for financial reasons has implication for the healthcare system, where out-of-pocket expenses account for a large portion of medical costs.

The mediating effect of unmet needs due to financial reasons accounted for $14.7 \%-32.9 \%$ of the TMEs of income. The effects were lower (7.2\%-18.7\%) in the lagged model, which is inconsistent with the proposal in previous studies that a time lag exists between the impact of low income on health status [28-30]. In other words, this result may imply that the time lag between unmet needs and the manifestation of self-rated poor health may not be as long as a year. Further studies will need to explore how long it takes for income level to affect self-rated health status and its mediated effects.

We acknowledge that this study has certain limitations. First, the issue of sequential ignorability assumption must be addressed to verify the causal relationship of the results of the mediating effect analysis. Parametric sensitivity analysis is known to be useful for this purpose [23]. However, in case where both the result variable and the mediator variable are dichotomous, we could not find currently available method to carry out a sensitivity analysis. Just as unmet needs affect poor health status, the converse may also hold; namely, poor health status may lead to increased medical needs. Studies have proven that more needs were unmet when self-rated health was consistently poor [31-34]. Future studies investigating this dynamic should be followed.

Second, attrition bias caused by follow-up loss was found during the process of compiling the panel data. The population who dropped out seemed to have distinctive characteristics, which should be reflected in the design of future studies. Nonetheless, by using an unbalanced panel for the 5-year period, this study reduced selection bias compared to previous 
studies $[20,35,36]$ that used a balanced panel that included those consistently participated in the survey over the entire study period.

Third, this study estimated the mediating effects of unmet needs on the impact of low income on health effects by using low income as the independent variable, not medical needs. This was based on the determination that low income is associated with health risks and susceptibility, poor health behaviors, and a high prevalence of diseases, meaning that it can be used as a proxy indicator for healthcare needs. Still, proximation of healthcare needs by low income brings various uncertainties that impede the estimation, insomuch as unmet need interacts with various variables in the model, which leads to the estimation of effect size that cannot be interpreted as causal inference by itself. Also, subjectively measured unmet needs used in the study may have broader meaning beside the unachieved healthcare need in clinical approach. Further research should be done to more comprehensively understand the mediating effects of unmet needs under the conceptual definition suggested in the introduction, especially on the roles that unmet needs play in the course of dealing with certain healthcare needs, and how they impact health in a qualitative way.

It is also crucial to note that the concept of unmet needs is a flexible notion with ambiguous definitions, both clinically and subjectively, although its intuitive and empirical quality is highly useful. As Levesque et al. [37] suggested in his model of patient-centered access to healthcare, healthcare needs may be unmet at any stage of the process of healthcare, ranging from perception of need to healthcare seeking, reaching, utilization, and health outcome. We must consider the qualitative dimension of unmet need as well as the traditional quantitative dimension in order to actually fulfil the need that makes people's health poor and suffer $[37,38]$. For example, Patients who report unmet healthcare need may seek alternative treatment such as supplementary remedies and supplementary product, including those that are not medically verified to be secure, which may deteriorate the continuity of care.

Lastly, though the result of lagged dependent variable model used in this study are not significantly different from crosssectional studies, it has certain limitations. The results may show trends for each year, but interactions among the different independent variables, outcome variables, and parameters each year were not dealt in the analysis. While this study fails to analyze the panel data in consideration with the serial cor- relation, several equations that can calculate mediating effects over years using changing variables over time have been recently suggested $[39,40]$. These methods are known for selection bias, sensitivity to confounding variables, and other disadvantages, but could enable a more comprehensive analysis of multi-year data suitable for the purposes of this study.

\section{SUPPLEMENTARY MATERIALS}

Supplementary material is available at https://www.jpmph. org/.

\section{CONFLICT OF INTEREST}

The authors have no conflicts of interest associated with the material presented in this paper.

\section{ACKNOWLEDGEMENTS}

This paper was based on the manuscript presented in 2017 Korean Health Panel Conference.

\section{ORCID}

Youngsoo Kim http://orcid.org/0000-0003-4769-2756

Saerom Kim http://orcid.org/0000-0002-1766-8432

Seungmin Jeong http://orcid.org/0000-0002-5409-3324

Sang Guen Cho http://orcid.org/0000-0002-9507-9983

Seung-sik Hwang http://orcid.org/0000-0002-1558-7831

\section{REFERENCES}

1. Donabedian A. Aspects of medical care administration: specifying requirements for health care. Cambridge: Harvard University Press; 1973, p. 65-68.

2. Huh SI, Kim SJ. Unmet needs for health care among Korean adults: differences across age groups. Korean J Health Econ Policy 2007;13(2):1-16 (Korean).

3. Shin YJ, Shon Jl. The prevalence and association factors of unmet medical need-using the 1st and 2nd Korea welfare panel data. Health Soc Welf Rev 2009;29(1):111-142 (Korean).

4. Alonso J, Orfila F, Ruigómez A, Ferrer M, Antó JM. Unmet health care needs and mortality among Spanish elderly. Am J Public Health 1997;87(3):365-370.

5. Lasalvia A, Bonetto C, Malchiodi F, Salvi G, Parabiaghi A, Tan- 
sella $M$, et al. Listening to patients' needs to improve their subjective quality of life. Psychol Med 2005;35(11):1655-1665.

6. Kortrijk HE, Kamperman AM, Mulder CL. Changes in individual needs for care and quality of life in Assertive Community Treatment patients: an observational study. BMC Psychiatry 2014; 14:306.

7. Park HJ, Park SS. The relationship between income level and health-related quality of life in Korea. Health Soc Sci 2012;31(1): 107-125 (Korean).

8. Park MJ, Choi SE. The effects of health behavior and health status on heath-related quality of life in older people: gender analysis by using the 2012 Korea Health Panel data. J Korean Acad Community Health Nurs 2017;28(2):118-128 (Korean).

9. Huh SI, Kim MG, Lee SH, Kim SJ. A study for unmet health care need and policy implication. Korea Inst Health Soc Aff 2009;5: 3-30 (Korean).

10. Heo J, Oh J, Kim J, Lee M, Lee JS, Kwon S, et al. Poverty in the midst of plenty: unmet needs and distribution of health care resources in South Korea. PLoS One 2012;7(11):e51004.

11. Cho SS, Lee TK, Bang YW, Kim CJ, Im HJ, Kwon YJ, et al. Factors associated with unmet needs for medical care among island inhabitants in Korea. J Agric Med Community Health 2010; 35(2):151-164 (Korean).

12. Park S, Kim B, Kim S. Poverty and working status in changes of unmet health care need in old age. Health Policy 2016;120(6): 638-645.

13. Marmot M. The influence of income on health: views of an epidemiologist. Health Aff (Millwood) 2002;21(2):31-46.

14. Lindahl M. Estimating the effect of income on health and mortality using lottery prizes as an exogenous source of variation in income. J Hum Resour 2005;XL(1):144-168.

15. Frijters $P$, Haisken-DeNew JP, Shields MA. The causal effect of income on health: evidence from German reunification. J Health Econ 2005;24(5):997-1017.

16. Aaberge R, Melby I. The sensitivity of income inequality to choice of equivalence scales. Rev Income Wealth 1998;44(4): 565-569.

17. Idler EL, Benyamini Y. Self-rated health and mortality: a review of twenty-seven community studies. J Health Soc Behav 1997; 38(1):21-37.

18. Idler EL, Angel RJ. Self-rated health and mortality in the NHANESI Epidemiologic Follow-up Study. Am J Public Health 1990; 80(4):446-452.

19. Woo HK, Moon OR. The difference of mortality according to self-assessed health status. Health Policy Manag 2008;18(4):
49-65 (Korean).

20. Ko H. Unmet healthcare needs and health status: panel evidence from Korea. Health Policy 2016;120(6):646-653.

21. Richiardi L, Bellocco R, Zugna D. Mediation analysis in epidemiology: methods, interpretation and bias. Int J Epidemiol 2013;42(5):1511-1519.

22. Preacher KJ. Advances in mediation analysis: a survey and synthesis of new developments. Annu Rev Psychol 2015;66: 825-852.

23. Imai K, Keele L, Tingley D. A general approach to causal mediation analysis. Psychol Methods 2010;15(4):309-334.

24. Imai K, Keele L, Tingley D, Yamamoto T. Unpacking the black box of causality: learning about causal mechanisms from experimental and observational studies. Am Polit Sci Rev 2011; 105(4):765-789.

25. Bynum B. The McKeown thesis. Lancet 2008;371(9613):644645.

26. Reeves A, McKee M, Mackenbach J, Whitehead M, Stuckler D. Public pensions and unmet medical need among older people: cross-national analysis of 16 European countries, 20042010. J Epidemiol Community Health 2017;71(2):174-180.

27. Karanikolos M, Gordeev VS, Mackenbach JP, McKee M. Access to care in the Baltic States: did crisis have an impact? Eur J Public Health 2016;26(2):236-241.

28. Blakely TA, Kennedy BP, Glass R, Kawachi I. What is the lag time between income inequality and health status? J Epidemiol Community Health 2000;54(4):318-319.

29. Kang YJ, Jung KH. An empirical study on income inequality and health in Korean society. Korean Public Adm Rev 2012; 46(4):265-291 (Korean).

30. Buddelmeyer $\mathrm{H}$, Cai L. Interrelated dynamics of health and poverty in Australia; 2009 [cited 2018 Oct 15]. Available from: http://ftp.iza.org/dp4602.pdf.

31. Idler EL, Kasl SV. Self-ratings of health: do they also predict change in functional ability? J Gerontol B Psychol Sci Soc Sci 1995;50(6):S344-S353.

32. Song KS, Lee JH, Rhim KH. Factors associated with unmet needs for health care. Korean Public Health Res 2011;37(1): 131-140 (Korean).

33. Shin J. Determinants of unmet healthcare needs among the community dwelling Korean adults with disabilities: a case of the National Health Insurance beneficiaries. Health Soc Welf Rev 2013;33(2):549-577 (Korean).

34. Shon C, Yi S, Hwang J. Factors affecting unmet healthcare needs among Korean migrants in Hong Kong. Korean Public 
Health Res 2015;41(1):107-121 (Korean).

35. Huh SI, Lee HJ. Unmet health care needs and attitudes towards health care system in Korea. Korean J Health Econ Policy 2016; 22(1):59-89 (Korean).

36. Lee HJ, Heo SI. Unmet health care needs and impact of type of household among the elderly in Korea. Korean J Health Econ Policy 2017;23(2):85-108 (Korean).

37. Levesque JF, Harris MF, Russell G. Patient-centred access to health care: conceptualising access at the interface of health systems and populations. Int J Equity Health 2013;12:18.
38. Kim DJ, Chae SM, Choi JH, Kim CY, Kim MH, Park YK, et al. Socioeconomic inequalities in health in Korea: magnitude of its challenges and strategy for alleviation (I). Sejong: Korea Institute for Health and Social Affairs; 2016, p. 357-358 (Korean).

39. Turnes PB, Ernst R. The Use of longitudinal mediation models for testing causal effects and measuring direct and indirect effects. China USA Bus Rev 2016;15(1):1-13.

40. VanderWeele TJ, Tchetgen Tchetgen EJ. Mediation analysis with time varying exposures and mediators. J R Stat Soc B 2017; 79(3): 917-938. 